\title{
3D texture-based face recognition system using fine-tuned deep residual networks
}

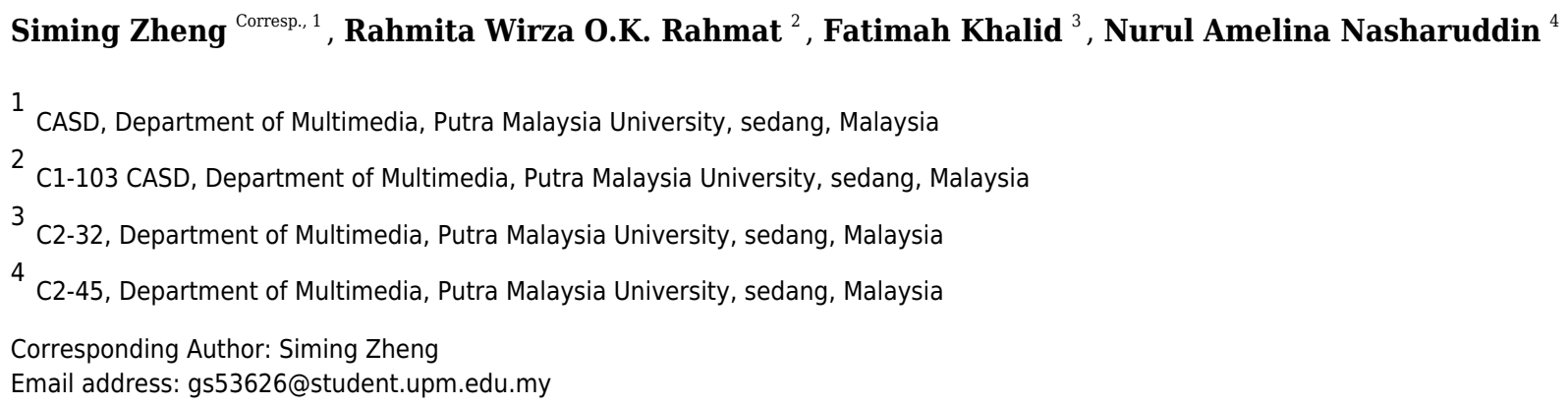

As the technology for 3D photography has developed rapidly in recent years, an enormous amount of 3D images has been produced, one of the researches for which is face recognition. Improving the accuracy of a number of data is crucial in the 3D face recognition problems. Traditional machine learning methods can be used to recognize 3D faces, but the face recognition rate has declined rapidly with the increasing number of 3D images. As a result, classifying large amounts of 3D image data is time-consuming, expensive, and inefficient. The deep learning methods have become the focus of attention in the 3D face recognition research. In our experiment, the end-to-end face recognition system based on 3D face texture is proposed, combining the geometric invariants, histogram of oriented gradients and the fine-tuned residual neural networks. The research shows that when the performance is evaluated by the FRGC-v2 dataset, as the fine-tuned ResNet deep neural network layers are increased, the best Top-1 accuracy is up to $98.26 \%$ and the Top- 2 accuracy is $99.40 \%$. The framework proposed costs less iterations than traditional methods. The analysis suggests that a large number of 3D face data by the proposed recognition framework could significantly improve recognition decisions in realistic 3D face scenarios. 
1 3D texture-based face recognition system 2 using fine-tuned deep residual networks

3

4 5 10

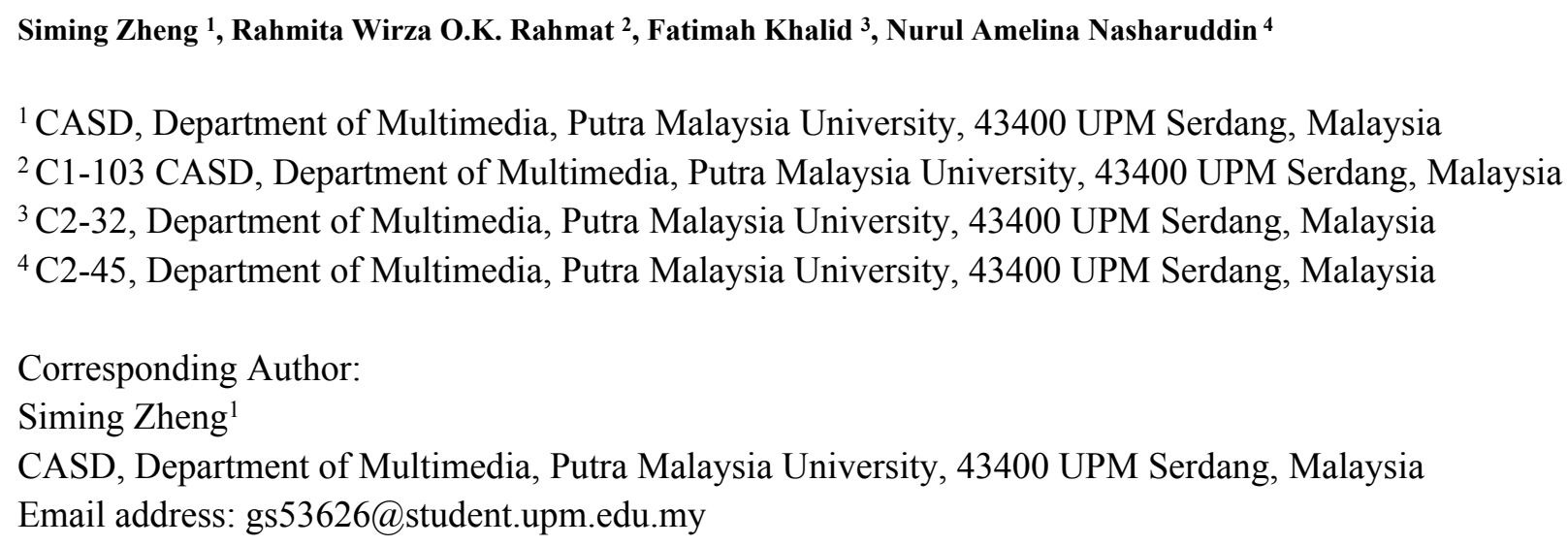

\section{ABSTRACT}

As the technology for 3D photography has developed rapidly in recent years, an enormous amount of 3D images has been produced, one of the researches for which is face recognition. Improving the accuracy of a number of data is crucial in the 3D face recognition problems. Traditional machine learning methods can be used to recognize 3D faces, but the face recognition rate has declined rapidly with the increasing number of 3D images. As a result, classifying large amounts of 3D image data is time-consuming, expensive, and inefficient. The deep learning methods have become the focus of attention in the 3D face recognition research. In our experiment, the end-toend face recognition system based on 3D face texture is proposed, combining the geometric invariants, histogram of oriented gradients and the fine-tuned residual neural networks. The research shows that when the performance is evaluated by the FRGC-v2 dataset, as the fine-tuned ResNet deep neural network layers are increased, the best Top- 1 accuracy is up to $98.26 \%$ and the Top-2 accuracy is $99.40 \%$. The framework proposed costs less iterations than traditional methods. The analysis suggests that a large number of 3D face data by the proposed recognition framework could significantly improve recognition decisions in realistic 3D face scenarios.

\section{Subjects Artificial Intelligence, Computer Vision.}

Keywords 3D textures, face recognition system, histogram of oriented gradients features, deep learning, residual neural networks, fine-tuning, Tensorboard.

\section{INTRODUCTION}


With the rapid development of the Internet, the smart computing equipment and social networking applications are increasingly used. There are hundreds of millions of 3D images uploaded every day to platforms such as Snapchat and Alipay, on which a large number of 3D face images are generated. Three main problems in creating 3D face recognition systems that many researchers report are the 3D face pose, illumination changes, and variations in facial expression. Extracting better features are a key process for 3D face recognition (Parama Bagchi et al., 2015; Jian Zhang et al., 2016; Gawed M. Nagi, 2013; Xueqiao Wang et al., 2015; Xiangyu Zhu et al., 2017). Furthermore, shallow learning (such as machine learning) including only one or no layer of hidden units leads to lack of ability to deal with large-scale data. These challenges have caused persistent problems for the robustness and reliability of such systems, which has driven many researchers to use deep learning for 3D face recognition tasks.

When deep learning methods are applied in realistic 3D face scenarios, two challenges confronted are as follows: Firstly, the accuracy becomes unstable as 3D face images are added this is because different deep learning networks have different generalization ability extracting images features. When processing a large number of image data, the deeper the layers of deep learning model are, the more problems such as gradient vanishing and gradient exploration will be caused; Secondly, as more and more complex deep learning models will be applied to the actual scenario, the recognition rate may be affected by the depth of a complex model. In this paper we explore both issues. How to recognize a large number of 3D face graphics with high precision is the main task of this paper.

In this work, the primary objective of approaches we proposed is to create an end-to-end face recognition system 3D textures-based with a high recognition accuracy, a satisfied performance and robustness while remaining practical. In this system, we have developed a residual neural network model base on ResNet for the 3D face recognition task. This model is fine-tuned with different depth using HOG featured 3D face textures. The primary aim is to solve problems of gradient vanishing and gradient exploration. We trained fine-tuned ResNet models with different depth using HOG based 3D texture images, maintaining faster calculations and the high accuracy of the growth of images.

The remainder of this work is prepared as follows. Section II reminds the related work. Section III presents methodology of extraction of HOG features and the fine-tuning ResNet model. Section IV shows the experimentation, results and discussion is described in Section V. The conclusions are finally stated in and VI. 


\section{7}

78

79

80

81

82

83

84

85

86

87

88

89

90

91

92

93

94

95

96

97

98

99

100

101

102

103

104

105

106

107

108

109

110

111

112

113

114

115

116

\section{RELATED WORKS}

Deep learning algorithms have received increasing attention in the face recognition field, and many researchers discovered the importance of studying 3D face recognition (Somsukla Maiti et al., 2014; Rui Min et al., 2012; Sebastian Pabiasz et al., 2017; Dania Porro-Munoz, et al., 2014; Huiying Hu et al., 2017; Y. Sun et al., 2015; Zhuoran Wu et al., 2017; Tang, H. et al.,2013; Delong Zhang et al.,2019). On one hand, extracting 3D face information is the key step in 3D face recognition: effective face detection and alignment can increase the overall performance of 3D face recognition, which is critical in both security and commercial 3D face recognition systems. On the other hand, researchers have proposed some methods for exploiting and exerting the deep learning for 3D face recognition, and they have demonstrated that the performance of deep learning systems is significantly better than that of machine learning method in the case of a large amount of $3 \mathrm{D}$ images.

In recent years, the convolutional neural network $(\mathrm{CNN})$ models have been used for 3D face recognition. Huiying Hu et al., (2017) has proposed a method of customizing convolutional neural networks. Her CNN's layer configuration uses the same principle to design based on the LeCun model (Y. LeCun et al., 1989). The structure of her model, called CNN-2, comprises one convolutional layer, one pooling layer, and a $5 \times 5$ filter. However, this structure cannot effectively extract and analyze $3 \mathrm{D}$ face data. When the learning rate rose from 0.034 to 0.038 , the classification accuracy increased from $84.04 \%$ to $85.15 \%$; while, the accuracy dropped to $81.31 \%$ when the learning rate rose to 0.042 . Furthermore, using a $7 \times 7$ filter increased the classification accuracy significantly to $84.75 \%$ with a learning rate of 0.034 .

In a follow-up study, S Sharma \& Sameera Shaik (2016) have proposed a new methodology for face recognition with a higher accuracy of approximately $98 \%$. They suggested a customized CNN model, including an input layer, a convolutional layer, a pooling layer, and a fully connected layer. They use the above method to recognize the $3 \mathrm{D}$ image with resolution of $96 \times 96$. According to results, their face recognition system takes twenty epochs for converging the learning rate, which includes the training rate and the testing rate. Especially, the training losses can be decreased to about 0 before the $6^{\text {th }}$ epoch.

Different methods have been proposed to recognize 3D face images. Donghyun Kim et al., (2017) has developed the VGGNet neural network for dealing with 3D face data. The most representative features of face are extracted from the fine-tuned VGGNet model. The model includes two convolution layers and two fully connected layers with random initial weights, using the last fully connected layer with a softmax layer to accommodate the different sizes of the input images. The fine-tuned VGGNet model achieved an accuracy of $95.15 \%$ in the experiment.

Gawed M. Nagi, (2013) has developed the face alignment algorithm based on the methods of geometric invariants, local binary pattern (LBP), and k-nearest neighbor (kNN). The face 
117 landmarks model (22 key points) is used to detect the human face, and the LBP method is used to 118 crop the 3D face areas. The method of $\mathrm{kNN}$ calculates the distance between each input data and 119 the training sample, obtaining the $\mathrm{k}$ images closest to the training sample. Finally, proposed 120 statistical methods are used to classify and recognize the images. The results show that the model

121

122

123

124

125

126

127

128

129

130

131

132

133

134

135

136

137

138

139

140

141

142

143

144

145

146

147

148

149

150

151

152

153

154

155 can reach $91.2 \%$ in the recognition rate; however, it declined to $84 \%$ as the number of datasets increases.

Sima Soltanpour et al., (2017) uses normal vector to study 3D face recognition. She proposed that more detailed distinct information can be extracted from the 3D facial image by using highorder LNDP method. By estimating the three components of normal vectors in $\mathrm{x}, \mathrm{y}$ and $\mathrm{z}$ channels, three normal component images are extracted. The score-level fusion of three high-order LNDP3x, LNDP3y and LNDP3z are used to improve the recognition performance. Experiments use SIFTbased strategy for matching the face features. The results of this study indicate that fusion LNDP3xyz outperforms descriptors, effectively improving the 3D recognition rate to $98.1 \%$.

The study by Patrik Kamencay et al., (2017) offers probably the most comprehensive empirical analysis of $3 \mathrm{D}$ face recognition. In an attempt to build practical and robust face recognition systems, he proposed three main types of layers for CNN architectures: the convolution layer, the pooling layer, and the fully connected layer. He also proposed three machine learning methods for face recognition, such as Principal Component Analysis (75.2\%), Local Binary Patterns Histograms (78.1\%), and $\mathrm{kNN}$ (71.5\%). The proposed customized $\mathrm{CNN}$ for 3D face recognition outperforms the above machine learning methods, which reaches the average accuracy of approximately $96.35 \%$. The highest accuracy is $98.3 \%$ when $80 \%$ of the data was used for training model.

Recent advances in HOG feature extraction methods have facilitated investigation of face recognition. In Geetika Singh \& Indu Chhabra's (2018) article, she suggests that HOG features can be used in the recognition system for improving the efficiency and the processing speed. The function of HOG features can capture the edge features that are invariant to the rotation and light. Owing to the fact that both texture and edge information is important for face representation. HOG features and SVM classifier-based face recognition algorithm is presented in Harihara Santosh Dadi \& Gopala Krishna Mohan Pillutla's (2016) research. His proposed model extracts the HOG features of the face from the image, these features can be given to any classifier. In the testing stage, the test image is obtained and fed to the SVM classifier, which is a non-probabilistic binary classifier and looks for optimal hyperplane as a decision function, for classification. The results show that this method has better classification accuracy for the test data set, about $92 \%$. In addition, compared to the method using standard eigen feature and PCA algorithm as a baseline, SVM also possesses an improved face recognition rate of $3.74 \%$. 
To investigate the effect of utilizing HOG features in the CNN model, Hafiz Ahamed et al., (2018) developed CNN learning models that using the HOG features as input data to the training model. His model contains of several layers and each layer is repeatedly used, finally a deep neural network is constructed. In order to evaluate the proposed model, a set of images with 160 images are generated for testing the model performance. However, it leads to a low generalization ability since the data set trained by the model is small. The result shows that the accuracy is approximately $89 \%$ by using the constructed model.

In the experiment, we used the latest residual deep neural network (ResNet) and the fine-tuning method (Kaiming He et al., 2015). Our preprocessing method uses HOG features of 3D face texture, different layers of ResNet are created during the experiment and whether decision making in face recognition process can be improved or not is investigated. We evaluated these approaches in the context of the same 3D face-recognition experiment as in (Patrik Kamencay et al., 2017), a more challenging task than the face identification task used in (Hafiz Ahamed et al., 2018).

\section{MATERIALS \& METHODS}

The diversity of face poses raises difficulties for 3D face recognition. By detecting key points on the face, such as the tip of the nose, the corners of the mouth, and the corners of the eyes, the face image in an arbitrary pose can be converted into a frontal face image by affine transformation, after which the face features can be extracted, and an identification is performed. This approach shows that after alignment the features can be extracted with greater success, and the recognition accuracy is thus greatly improved. A schematic diagram of face detection and face alignment is shown in Figure 1. There are three steps for preprocessing of 3D face recognition: 1. 3D face detection, 2. 3D face alignment. 3. 3D human face feature extraction. The first two phases are implemented by uses the open-source tool provided by the Dlib, which can monitor the key points of the face real time to obtain the position and posture of the face. Then we developed a module for extracting the HOG features based on 3D face texture images. Key points of the face are detected using the conditional local neural fields algorithm (Tadas Baltrusaitis et al., 2013; Karen Simonyan and Andrew Zisserman. 2015).

\section{A. Facial Detection and Landmarks Selection}

\section{Figure 2 Facial landmarks (68 key points) of Face Recognition Grand Challenge Version 2(FRGC v2.0).}

All 3D images need to be processed before the processing of recognition in order to reduce image noise and redundancy, as shown in Figure 2. The first step of 3D face recognition is face detection 
195

196

197

198

199

200

201

202

203

204

205

206

207

208

209

210

211

212

213

214

215

216

217

218

219

220

221

222

223

224

225

226

227

228

229

230

and alignment. We use pre-trained facial landmark detector from the Dlib library, which is used to estimate and predict the location of sixty-eight key points on the human face.

Based on the geometric invariant method, these facial points are marked on the 3D facial images (Tadas Baltrusaitis et al., (2016); Amin Jourabloo \& Xiaoming Liu (2015); Amanda Song et al., 2017), the subgraphs of $A$ and $C$ is the original 3D images, and the 68 key point distributions are indicated as B and D in Figure 2 on the right side. These points, including the dominant facial features, such as the tip of the nose, the corners of the mouth, and the corners of the eyes, which are used for further feature extraction and geometric calculations in the recognition stage.

\section{B. The features of Histogram of Oriented Gradient}

In the feature extraction process, we usually try to find the invariant properties and characteristics so that the extraction results do not change significantly due to the specified conditions, this means that the goal of recognition is to find useful discriminative information not the position and size. Regardless of the different changes in the shape and appearance of the image, we should find reliable and robust discriminative information for improve the recognition rate.

In the field of image processing and computer vision, texture analysis and extraction have a rich history. A method called the Histogram of Oriented Gradient (HOG) has received extensive attention. The core idea of the HOG method is to describe the texture of the detected object by the gradient or distribution of edge directions. Its function is to capture the edge or gradient structure from the image, which is characteristic for the representation of local texture. The benefit of this feature is relatively less affected by the appearance and shape. Essentially, it forms a template and uses learning models to effectively promote recognition.

The HOG descriptor can extract important features from 3D images (Harihara Santosh Dadi \& Gopala Krishna Mohan Pillutla' 2016; Pranav Kumar et al., 2016). It captures the local texture information well and has good invariance to geometric and optical changes. Firstly, the target image is divided into small connected regions, which call the cell units. Then, the gradient or edge direction of each pixel in the cell unit are acquired. Finally, the histograms can be combined to form a feature descriptor. In this section, the HOG feature is used as a means of feature extraction in the process of recognition, the purpose is to combine the discriminative $3 \mathrm{D}$ face feature in the recognition phase, the specific implementation steps are as follows.

\section{1) Color and gamma normalization}

To reduce the influence of lighting factors, the entire image needs to be normalized in the first step. A compressing process that can effectively reduce shadows, colors and illumination variations of the image, because this information did greatly increase code complexity and 
231

232

233

234

235

236

237

238

239

240

241

242

243

244

245

246

247

248

249

250

251

252

253

254

255

256

257

258

259

260

261

262

263

264

265

266

267

268

demanded the higher performance of processor. At the same time, the gray image is normalized by Gamma formula. By smoothing part of noises, the influence of local strong light on gradient calculation is reduced. The $\gamma$ is the symbol of Gamma and its value is 1 . The formula of gamma compression (equation 1) is shown below.

$$
I(x, y)=I(x, y)^{\gamma}
$$

\section{2) Gradient computation}

The gradient value of each pixel position is calculated in this step. The derivation operation can capture contours, human shadows, and some texture information, which further weakens the influence of illumination. In the operation of computing image gradient, the gradient direction is key to HOG algorithm. The function of $H$ is used for calculating of Histogram of Oriented Gradient. Each pixel point of the transverse gradient $G_{x}(x, y)$ and the longitudinal gradient of the

$$
\begin{aligned}
& G_{x}(x, y)=H(x+1, y)-H(x-1, y) \\
& G_{y}(x, y)=H(x, y+1)-H(x, y-1)
\end{aligned}
$$

3) Creating the orientation histograms

The algorithm needs to finish some operations that calculating the direction gradient of the smallest interval. At the beginning, the 3D image is divided into several intervals with different sizes. Starting from the smallest interval, the gradient direction of all the pixels are contained in each interval, which are weighted by the magnitude. The gradient direction with the largest value represents the gradient direction in the current interval. Finally, the gradient magnitude $G(x, y)$ of each pixel point is calculated according to equation 4 .

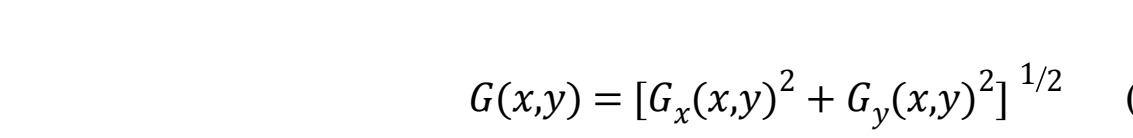

Furthermore, the specific operation in the equation above is that the $G(x, y)$ ranges from $-90^{\circ}$ to $90^{\circ}$. Vectors can be evenly divided into nine intervals because each interval is $20^{\circ}$. This means that nine intervals consist of a total of nine feature vectors in a cell. Four cells can form a block, so each block includes 36 feature vectors. In this way, the feature vectors of all cells in a block are concatenated to form the HOG features. 
269

270

271

272

273

274

275

276

277

278

279

280

281

282

283

284

285

286

287

288

289

290

291

292

293

294

295

296

297

298

299

300

301

302

303

304

305

306

307

4) Computing the directional gradient histogram

In the final step of merging processing, the algorithm combines all blocks order by weighted voting from small to large. In some cases, the algorithm eliminates some detailed features, which are represented by the small amplitude gradient in the intervals. The rest of blocks are merged into a maximum gradient pattern, in which contains the important representative features in the 3D images. Gradient direction a $(\mathrm{x}, \mathrm{y})$ of the pixel point are calculated according to equation 5 .

$$
\alpha(x, y)=\tan ^{(-1)}\left[G_{y}(x, y) / G_{x}(x, y)\right]
$$

5) Creating the orientation histograms

After completing above works, the algorithm generated an orientation histogram for the input 3D face image. In this experiment, we make the pixel of $16 \times 16$ constitute a block in an image with $224 \times 224$ image, the stride of scanning window is $8 \times 8$, then there are 27 scanning windows in the horizontal and vertical directions in a 3D texture image. Therefore, each 3D texture image ( $36 \times 27 \times 27$ ) has 26244 dimensional vectors that can form a complete edge orientation histogram.

After the preprocessing, the face image with extracted HOG features of 3D textures is input into our fine-tuned ResNet classification model. The information contained in the original image is compressed and adjusted, which greatly improves the performance of the subsequent feature extraction network in ResNet neural network. Finally, the whole processing of HOG feature extraction for 3D face image is shown in the Figure 3(A, B and C).

\section{Figure 3 The processing of HOG feature extraction.}

We also demonstrate generation of HOG feature vectors for specific person with different expression, scenarios and various illumination changes. The images based on HOG features extraction are shown from the $\mathrm{F}$ to J, which are separately correspond to the reprocessing aligned images from $\mathrm{A}$ to $\mathrm{E}$ in Figure 4.

\section{Figure 4 The HOG features of various 3D face images.}

\section{The architecture of ResNet neural networks}

Convolutional neural networks with multiple layers have several advantages in the research of image classification. The deep network uses a form of end-to-end neural network that automatically integrates the low, medium, and high-level features, and then transmits all of these 
308 features to the classifier, extracting different depth features by stacking layers with different

309

310

311

312

313

314

315

316

317

318

319

320

321

322

323

324

325

326

327

328

329

330

331

332

333

334

335

336

337

338

339

340

341

342

343

344

345

346

\section{Figure 5 The architecture of ResNet model.}

In general, the deeper the neural network is, the worse the recognition performance will be. One major issue in early 3D face recognition research is caused by the use of an error back-propagation algorithm (Steve Lawrence \& C. Lee Giles. 2000), which includes the weight coefficient, the derivative of the activation function, and the activation value in the partial derivative. When the number of layers is large, these values are multiplied, easily leading to the vanishing gradient and exploding gradient problems (Razvan Pascanu et al., 2012; Boris Hanin. 2018). Therefore, it is difficult to ensure high-accuracy in the case of growth of 3D face data. In the paper by Kaiming He et al., (2015), he proposed a theory of deep residual learning, which adopted an approach of shortcut connection to avoid the issues mentioned above. The ResNet architecture for a 152-layer network (a) and a residual block (b) are shown in Figure 5 above. A residual block with a connections layer can skip a specific layer in the network. The advantages of short connections are that it can reduce the problem of gradient disappearance, thus making the network converge faster and reducing parameters. ResNet-152 also uses the batch normalization operation between each convolution and activation. It allows the researcher to build increasingly deep networks, which have high recognition abilities.

\section{The fine-tuned ResNet neural network model}

In deep neural networks, the function of the first layer of training on images is similar to the Gabor filters and color spots operations. Such first-layer features are not used to be specific to specific data sets or specific tasks, but to general ones, because they are applicable to common data sets and tasks. Image features are eventually transmitted from general to specific by the last layer of the network (Jason Yosinski, et al., 2014). In the big data scenario, we introduce the fine-tuning method in the ResNet neural network, which can greatly shorten the training time, efficiency improve the training loss, and have a stronger generalization ability for getting a good result.

\section{1) Fine-tuning method}

The fine-tuning method can be used to flexibly adjust the architecture of the ResNet model in this 3D face recognition task (Heechul Jung et al., 2015). In our experiment, four pooling layers with the adaptive average pooling method have been reconstructed. By using the new architecture, it 
347 makes the input training data adaptive to the fine-tuned ResNet Model, and the computational

348 complexity of the model is reduced. A softmax layer is created after the fully connected layer to

349

350

351

352

353

354

355

356

357

358

359

360

361

362

363

364

365

366

367

368

369

370

371

372

373

374

375

376

377

378

379

380

381

382

383

384 implement the target data classification task of this experiment.

\section{2) Rectifier Linear Unit}

The Rectifier Linear Unit (ReLU) is an important activation function in the ResNet structure, which can overcome the problem of gradient disappearance and speed up the time of training (Abien Fred M. Agarap. 2018). A ReLU function maps the input value $x$ to 0 if it is negative, and keeps its value unchanged if it is positive, the main ReLU calculation expression (equation 6) is shown below.

$f_{\text {relu }}(x)=\max (0, x)$

The convolutional neural network architecture is mainly followed with a combination of all the methods described above. The architecture starts from the input layer of training images and is followed by the convolution layer with the optimum weight and bias for the feature layer. In order to reduce the internal covariate shift (Sergey Ioffe \& Christian Szegedy. 2015) in the deep neural network, the batch normalization algorithm is also added to each convolutional layer to perform the operations of normal normalization and affine transformation on the input of each layer. Finally, our fine-tuned ResNet model were constructed with the proposed method, and the parameters of each convolutional layer are represented in Figure 6.

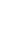

\section{Figure 6 The fine-tuned ResNet neural network.}

In this fine-tuned ResNet model, the layer of adaptive average pooling emphasizes the downsampling of the overall feature information, its purpose is to reduce the dimension of the feature and retain the effective information, it can integrate features in the feature maps from multiple convolutional layers and pooling layers so that the integrity of the information in this dimension can be more reflected. Through this process, both high-dimensional features and confidence scores can be obtained from each classification. The final full connection layer is used to synthesize the features extracted from the adaptive average pooling, it can output the probability distribution by using the softmax regression method, which can be divided into more than 1,000 classifications for any tasks, and the value of this parameter was set to 466 in our experiment. In the above structure, Adam function is used as an alternative to the traditional Stochastic Gradient Descent (SGD) optimization algorithm which can iteratively update the weights based on the training data mainly to optimize the neural network and make the training faster. 


\section{E. Datasets}

387

388

389

390

391

392

393

394

395

396

397

398

399

400

401

402

403

404

405

406

407

408

409

410

411

412

413

414

415

416

417

418

419

420

421

422

423

This research received the approval from the University of Notre Dame (henceforth, UND), and the dataset of Face Recognition Grand Challenge version 2 (FRGC-v2) in January 2019. This experiment was performed on FRGC-v2 (Patrick Flynn. 2006), which is a large number standard face image dataset containing over 50,000 high-resolution 2D and 3D face images, which divided into training and validation partitions in a laboratory setting. Training partition is designed for training algorithms, and validation partitions is used to evaluate the performance of a method in a laboratory environment. All the images were captured by a Minolta Vivid 900/910 series scanner. The datasets used belong to Experiment 3 of FRGC-v2. The experimental 3D face dataset includes 4,007 images of 466 people with different lighting and facial expressions (). The aim of this dataset is to test the ability to run experiments on large datasets (P.J. Phillips et al., 2005).

\section{F. Graphics Processing Unit}

The efficient parallel computing of the graphics processing unit (GPU) makes up for the slow training of deep neural networks (Zhilu Chen et al., 2014). Combined with the CUDA parallel computing platform, it allows the use of larger training datasets and deeper complex neural networks to extract deeper image features (Yi-bin Huan et al., 2015; Srishti Singh et al., 2017). The model of GPU used in this experiment is NVIDIA GeForce GTX 1080Ti. Its multi-core architecture includes thousands of stream processors, which can perform vector operations in parallel and achieve several times greater throughput in the application. This significantly shortens the calculation time. GPU has therefore been widely used by scientists in deep network learning.

\section{Figure 7 The fine-tuned ResNet feature extraction operation.}

The Figure 7 shows the processing of our proposed framework. The detailed steps of the proposed framework are as follows: First, the image is detected by the 68 key points of facial landmarks method, and the main multi-channel 3D face regions are extracted, this preprocessing module achieved precision rate of $99.85 \%$ and effectively reduces image noise and redundancy from original images, and all images are rescaled to the $224 \times 224 \times 3$. Then, the edges and textures of 3D face images are enhanced by using the HOG method with custom parameters, the HOG face feature images based on 3D textures are obtained, which learned higher discriminative features from 3D face images. Next, fine-tuned deep residual model is proposed by using the HOG textures as the input images. Finally, we generated custom ResNet neural network model. Its image input size is adjusted to the pixel of $224 \times 224 \times 3$, and the structure and quantity of the middle layer in the model are reconstructed, all the operations are performed by using fine-tuning method. 


\section{EXPERIMENT}

425 Key point detection and alignment were carried out on the 3D raw face texture in succession. In 426 the following steps, the dataset applied to the fine-tuned ResNet model comprises 3D face texture

427

428

429

430

431

432

433

434

435

436

437

438

439

440

441

442

443

444

445

446

447

448

449

450

451

452

453

454

455

456

457

458

459

460

461

60 with HOG features. Finally, the proposed model conducted for 3D face recognition. In this experiment, an implementation of GPU accelerated training is adopted based on Python and the CUDA architecture, all the HOG-featured images were resized of $224 \times 224 \times 3$ pixel in the dataset, the test model of fine-tuned ResNet with different depth layers (e.g., 50 layers, 101 layers, 152 layers) were then evaluated.

1) Firstly, a convolution layer in fine-tined ResNet architecture multiplies the $2 \times 2$ filter with a highlighted area (also $2 \times 2$ ) of the input feature map, and all the values are summed up to generate one value in the output feature map, as shown in Figure. 7.

2) After the 3D data are processed through the first convolution layer, the next layer is maxpooling. The filter window of the max-pooling is moved across the input feature with a step size defined by the stride (the value of stride is 2 in the case of ResNet-152).

The advantage is that it can reduce errors and preserve more texture information. In the maxpooling, the maximal value is selected from four values in the filter window. The size of the detection region is $f \times f$, with a stride of $s$, so the output features $h$ ' and $w^{\prime}$ are given through the equation 7 below.

$$
h^{\prime}=\left[\frac{h-f+s}{s}\right], \quad w^{\prime}=\left[\frac{w-f+s}{s}\right] .
$$

3) The residual block consists of two convolution layers each a $1 \times 1$ filter and one convolution with a $3 \times 3$ filter. The $1 \times 1$ layer mainly reduces and restores dimensions, leaving the $3 \times 3$ layer a bottleneck with smaller input/output dimensions. Two $1 \times 1$ convolutions effectively reduce the number of convolution parameters and the amount of calculation. The residual block is used for ResNet-50/101/152.

4) The fine-tuned ResNet model uses a global average pool and then categorizes 3D face images at the end of the network through fully connected layers. The global average pooling layer provides faster calculations with more accurate classification and fewer parameters. It serves to sum up all the values from the filter window and then average them, which can reduce errors and retain 3D background information of the image.

5) Finally, the fully connected layer reassembles the previous local 3D features into a complete graph through the weight matrix. The classification $y$ is defined as follows:

$$
y=f\left(W^{T} x+b\right)
$$


462

463

464

465

466

467

468

469

470

471

472

473

474

475

476

477

478

479

480

481

482

483

484

485

486

487

488

489

490

491

492

493

494

495

496

497

498

499

500

6) However, having a good neural network model in specified dataset does not necessarily imply that the model is perfect or that it will be reproduced when tested on external data. In order to make sure it is robust, reproducible and unbiased for testing future new datasets under non-ideal conditions, accuracy metrics is adopted to evaluate the fine-tuned ResNet model's performance.

The indicator accuracy is a measurement of the correct proportion of image classifications. This study is accurate in its ability to differentiate the $3 \mathrm{D}$ face recognition cases correctly. To estimate the accuracy of tests, the proportion of true positives (TP) and true negatives (TN) in all evaluated cases are calculated in this experiment. Mathematically, this can be stated as following.

$$
\text { Accuracy }=\frac{T P+T N}{T P+T N+F P+F N}
$$

The sub formula of TP+TN+FP+FN is the total number of observations. Moreover, the respective tests of Top 1 and Top 2 accuracy are used to evaluate the performance of our proposed model. The following hypothesis will be tested: The increasing network depth improves the accuracy of 3D face recognition. This study contributes to this developing area of research by exploring how different depth of our fine-tuned ResNet networks affect the outcome of 3D recognition.

\section{RESULTS AND DISCUSSION}

Tensor Board is a data visualization tool that can be used to visualize computational graph structure, provide statistical analysis, and plot the values captured as summaries during the execution of computational graphs. In this research, different types of pre-trained ResNet neural network with the same structure but different depths are proposed. As shown in Figure 8, the threesubgraph shown below are the Tensor Board graph of linear regression corresponding to the 50 (A), 101 (B), and 152 (C) layers of structure of the ResNet neural networks. The 3D face recognition rates of the three different structural models were recorded objectively in real time use of Tensor Board.

Figure 8 The accuracy rate of different layer numbers in the fine-tuned ResNet architecture. The performances are shown for fine-tuned ResNet-50 layer(A), fine-tuned ResNet-101 model(B), and Fine-tuned ResNet-152 model(C).

There were 21 times of training and testing in all test cases. According to the results of the ResNet50 testing model, the maximal accuracy was $97.02 \%$ for the validation set in the $21^{\text {th }}$ epoch, which corresponds to the similar accuracy of $97.22 \%$ in the $6^{\text {th }}$ epoch of the ResNet101 model. This accuracy rate is close to $97.10 \%$ in the same epoch in the ResNet152 model. The highest accuracies were $98.05 \%$ for ResNet 101 and $98.26 \%$ for ResNet 152 .

The most accurate indicator of Top 2 can be used to further evaluate the performance of the trained ResNet model. In the ResNet 152 model, the recognition rate fluctuates at first and then

Peer] Comput. Sci. reviewing PDF | (CS-2019:06:38908:1:3:NEW 6 Oct 2019) 
501 becomes regular with the increase of test sets. The accuracy rate was maintained at an average of

502

503

504

505

506

507

508

509

510

511

512

513

514

515

516

517

518

519

520

521

522

523

524

525

526

527

528

529

530

531

532

533

534

535

536

537

538

539

$99.30 \%$ for ResNet 152 after the $12^{\text {th }}$ epoch. The results show that the ResNet model has strong generalization ability. The recognition rate reaches its peak of $99.40 \%$ in ResNet- 152 in the $12^{\text {th }}$ epoch of the 3D face recognition experiment.

This experiment explores the benefits and effect of different numbers of neural network layer through the fine-tuning method on 3D face texture recognition research with high accuracy. The Figure 8 has shown that the increasing of layers of fine-tuned ResNet neural network model, the proposed framework can improve the accurate through the HOG method based on 3D face textures.

To eliminate the effects of interference factors, the 3D face dataset was processed beforehand (3D face detection, alignment, and HOG feature extraction). Studies have shown the importance of the fine-tuned convolutional neural network model (ResNet) with depth layers that have more highly discriminative features. The model is advantageous in that although the depth is significantly increased, the ResNet model is less complex with a higher accuracy rate. The Figure 8 presents the inter-correlations among the three recognition rates of the ResNet model with different layers. The 3D face recognition rate is positively correlated with the number of layers in the ResNet model, which is also a principal factor determining the computing time.

With qualitative modes of enquiry employed, the experiments show that the proposed method achieves promising results, demonstrating that the ResNet-152 neural network model described in this paper can have a recognition accuracy of $98.26 \%$ (Top 1); compared with the most accurate, the accuracy of the second accurate test was improved by $1.14 \%$ (at 99.40\%) with the FRGC-v2 datasets. Practical results proved the validity of the proposed method in 3D face recognition.

The classification performance of methods applied to the FRGC-v2 dataset seem superior to the seemingly impressive results of published studies utilizing different methods in Table 1 (Huiying Hu et al., 2017; S Sharma \& Sameera Shaik (2016); Sima Soltanpour \& Q.M. Jonathan Wu. 2017).

In previous researches, custom $\mathrm{CNN}$ is a commonly used deep learning algorithm used for 3D image recognition tasks. Firstly, in custom CNN network training, it is necessary to constantly adjust network parameters. The customized parameters such as weights and biases in CNN network results in a very slow convergence of training, and thus greatly increasing the training time and the number of epochs (Huiying Hu et al., 2017; S Sharma \& Sameera Shaik 2016). In addition, when the dimension increases with the increase of data volume, it will lead to a curse of dimensionality problems and cause a drop in the performance of the classifier (Sima Soltanpour \& Q.M. Jonathan Wu. 2017). The Fine-tuning method speeds up the convergence and shortens the training period, thus adapting to $3 \mathrm{D}$ processing. The purpose of multi-layer convolution is that the features acquired through one-layer convolution are often local, and the more layers there are, the

Peer) Comput. Sci. reviewing PDF | (CS-2019:06:38908:1:3:NEW 6 Oct 2019) 
540 more global the features will be acquired. Then, how to maintain good performance and improve 541 accuracy is a key in larger numbers of 3D face recognition scene. Therefore, fine-tuning depth 542 residual network is proposed based on HOG features to effectively solve the problem of large 543 numbers of 3D face recognition.

544

545

546

547

548

549

550

551

552

553

554

555

556

557

558

559

560

561

562

563

564

565

566

567

568

569

570

571

To the best of our knowledge, our work is to examine a fine-tuned Deep Residual Networks model on the recognition task of FRGC-v2 dataset. To increase accuracy during ResNet training, several methods were considered in this paper: 1) a fine-tuning deep residual network was adopted, taking advantage of its intrinsic features, such as shortcut connection, weights sharing and pooling architectures, and these can be improved through the deepening of the network structure; 2) the number of layers is carefully designed with smaller filter size to avoid overfitting while there is sufficient capacity for the network to solve the complex large number classification problems(Douglas M. Hawkins. 2004; Steve Lawrence and C. Lee Giles. 2000).; 3) data extraction was performed via the HOG method at the image preprocessing that contains higher discriminative features in the 3D images. As a result, the proposed methods were well trained and yielded stateof-the-art classification accuracy.

\section{TABLE 1 Performance comparisons between the proposed method and state-of- the-art methods based on the FRGC-v2 dataset.}

\section{CONCLUSIONS}

In this study, in-depth investigations were conducted on an end-to-end 3D face textures recognition. We first review the previous studies on 3D face recognition and then summarize the critical research questions to be solved. The 3D face detection and alignment modules are implemented and flexibly applied in 3D face raw data, which achieves a precision rate of $99.85 \%$. In addition, the detailed steps of the HOG extraction pattern were presented. 3D face images with HOG features can significantly minimize the descriptor size for reducing computation load and economizing the memory in the recognition process. We trained the fine-tuned ResNet models combined with HOG features, the discriminative power of the deeply learned features can highly enhance recognition ability. This study implemented every important subcomponent, which can effectively reduce 3D image noise, and greatly increase the robustness of our proposed recognition system.

572

573

The experiment showed that the degradation problem was efficiently solved by increasing the

574 number of layers in our fine-tuned ResNet neural networks, which improves the recognition rate

575 within a short time, and the accuracy is maintained at a certain level. However, the performance

576 of the algorithm is unexceptional in practical application, we think that several aspects of the model

577 should still be studied and improved. Firstly, although the HOG algorithm is advantageous in less

578 calculation time and faster detection speed, when the pose of the 3D face is changed drastically, 
579 there is a target loss in the face image, which leads to low processing efficiency. The 3D face 580 alignment can be pre-processed with the CNN detection method, and a multi-processing or 581 multithreading method can be used to speed up face alignment, which ensures that the pre582 processing module can process data quickly. Secondly, the recognition rate may be adversely 583 affected by certain conditions. For instance, the ResNet-152 model exhibited the phenomenon of 584 overfitting, in which the accuracy rate dropped and remained at around $97 \%$ after the $9^{\text {th }}$ epoch. 585 This phenomenon is caused by two conditions: too few datasets and the excessive complexity of 586 the neural network model. This can be solved by increasing the amount of $3 \mathrm{D}$ face data in the 587 future works via a data augmentation method (L Perez \& J Wang. 2017; SC Wong, 2016). This 588 also shows that the ResNet network has a more powerful data processing capability for a large

589

590

591

592

593

594

595

596

597

598

599

600

601

602

603

604

605

606

607

608

609

610

611

612

613

614

615

616 number of data. Overall, the development of large number 3D face recognition classification system is a challenging work, and there is still a long way to go to apply these theories and methods in large-scale scenes. The results suggest that fine-tuned deep residual networks classification approach based on HOG features will be a promising direction to improve 3D face recognition rate.

\section{ACKNOWLEDGEMENTS}

I am indebted to Prof. Rahmita Wirza O.K. Rahmat for assistance with data collection, providing helpful discussions of the 3D face analyses. I thank Fatimah Khalid for her help in the depth analysis and comments on the face recognition theory, as well as Prof. Dr. Nurul Amelina Nasharuddin for fruitful advices.

\section{REFERENCES}

Parama Bagchi, Debotosh Bhattacharjee, and Mita Nasipuri. 2015. 3D Face Recognition using surface normal. TENCON 2015 IEEE DOI 10.1109/TENCON.2015.7372819.

Jian Zhang, Zhenjie Hou, Zhuoran Wu, Yongkang Chen, and Weikang Li. 2016. Research of 3D Face Recognition Algorithm Based on Deep Learning Stacked Denoising Autoencoder Theory. ICCSN DOI 10.1109/ICCSN.2016.7586606.

Gawed M. Nagi, Rahmita Rahmat, Muhamad Taufik, and Fatimah Khalid. 2013. Multimodal 2D-3D Face Recognition. IJFCC DOI 10.7763/IJFCC.2013.V2.253.

Xueqiao Wang, Qiuqi Ruan, Yi Jin, and Gaoyun An. 2015. 3D face recognition using closest point coordinates and spherical vector norms. ICWMMN DOI 10.1049/cp.2015.0943. 
617 Somsukla Maiti, Dhiraj Sangwan, Jagdish Lal Raheja. 2014. Expression-Invariant 3D Face

618 Recognition Using K-SVD Method. Applied Algorithms. 8321:268-275.

619

620

Rui Min, Jongmoo Choi, Gérard Medioni, and Jean-Luc Dugelay. 2012. Real-time 3D face

621 identification from a depth camera. ICPR DOI 10.1109/CRV.2018.00020.

622

623

Sebastian Pabiasz, Janusz T. Starczewski, and Antonino Marvuglia. 2015. SOM vs FCM vs

624 PCA in 3D Face Recognition. Artificial Intelligence and Soft Computing 9120:120-126.

625

626

Dania Porro-Munoz, Francisco Jose Silva-Mata, Anier Revilla-Eng, Isneri Talavera-

627 Bustamante, and Stefano Berretti. 2014. 3D Face Recognition by Functional Data Analysis.

628

Progress in Pattern Recognition, Image Analysis, Computer Vision, and Applications 8827:819-

629 826.

630

631

Huiying Hu, Syed Afaq Ali Shah, Mohammed Bennamoun, and Michael Molton. 2017. 2D

632 and 3D Face Recognition Using Convolutional Neural Network. TENCON 2017 IEEE DOI

633 10.1109/TENCON.2017.8227850.

634

635

Y. LeCun, B. Boser, J. S. Denker, D. Henderson, R. E. Howard, W. Hubbard, and L. D.

636

Jackel. 1989. Backpropagation applied to handwritten zip code recognition. Neural Computation

637 1:541-550.

638

639

S Sharma and Sameera Shaik. 2016. Real Time Face Authentication Using Convolutional 640

Neural Network. International Conference on Signal Processing(ICSP) DOI 641 10.1049/cp.2016.1455.

642

643

Donghyun Kim, Matthias Hernandez, Jongmoo Choi and Gerard Medioni. 2017. Deep 3D

644 Face Identification. 2017 IEEE International Joint Conference on Biometrics (IJCB) DOI 645 10.1109/BTAS.2017.8272691.

646

647 Patrik Kamencay, Miroslav Benco, Tomas Mizdos, Roman Radil. 2017. A New Method for 648 Face Recognition Using Convolutional Neural Network. Digital Image Processing and Computer 649 Graphic 15:664-670.

650

651

Tadas Baltrusaitis, Peter Robinson, and Louis-Philippe Morency. 2013. Constrained Local 652 Neural Fields for Robust Facial Landmark Detection in the Wild. ICCV, DOI 653 10.1109/ICCVW.2013.54.

654 
655 Karen Simonyan and Andrew Zisserman. 2015. Very Deep Convolutional Networks for Large656 Scale Image Recognition. 2015 3rd IAPR Asian Conference on Pattern Recognition (ACPR) DOI 657 10.1109/ACPR.2015.7486599.

658

Christian Szegedy, Wei Liu, Yangqing Jia, Pierre Sermanet, Scott Reed, Dragomir 660 Anguelov, Dumitru Erhan, Vincent Vanhoucke, and Andrew Rabinovich. 2014. Going 661 Deeper with Convolutions. 2015 IEEE Conference on Computer Vision and Pattern Recognition. DOI 10.1109/CVPR.2015.7298594.

663

664

Tadas Baltrusaitis, Peter Robinson, and Louis-Philippe Morency. 2016. OpenFace: an open 665 source facial behavior analysis toolkit. IEEE Winter Conference on Applications of Computer Vision DOI 10.1109/WACV.2016.7477553.

667

Harihara Santosh Dadi and Gopala Krishna Mohan Pillutla. 2016. Improved Face 669 Recognition Rate Using HOG Features and SVM Classifier. IOSR Journal of Electronics and 670 Communication Engineering 11:36-37.

671

Pranav Kumar, S L Happy, and Aurobinda Routray. 2016. A Real-time Robust Facial 673 Expression Recognition System using HOG Features. International Conference on Computing, 674 675 Analytics and Security Trends DOI 10.1109/CAST.2016.7914982.

677

678

679

Razvan Pascanu, Tomas Mikolov, and Yoshua Bengio. 2012. Understanding the exploding gradient problem. arXiv 1211.5063:3-4.

680

Boris Hanin. 2018. Which Neural Net Architectures Give Rise to Exploding and Vanishing 681

682 Gradients? 32nd Conference on Neural Information Processing Systems (NeurIPS) 1:580-589.

683

Kaiming He, Xiangyu Zhang, Shaoqing Ren, and Jian Sun. 2015. Deep Residual Learning for 684 Image Recognition. IEEE Conference on Computer Vision and Pattern Recognition (CVPR) DOI

685

686 10.1109/CVPR.2016.90.

Sergey Ioffe and Christian Szegedy. 2015. Batch normalization: Accelerating deep network 687 training by reducing internal covariate shift. International Conference on Machine Learning 688 37:2-5.

689

690

Abien Fred M. Agarap. 2018. Deep Learning using Rectified Linear Units (ReLU). arXiv 691 1803.08375v2: $1-6$.

692 
693 Zhilu Chen, Jing Wang, Haibo He, and Xinming Huang. 2014. A Fast Deep Learning System 694 Using GPU. IEEE International Symposium on Circuits and Systems (ISCAS) DOI 695 10.1109/ISCAS.2014.6865444.

696

697

Sima Soltanpour and Q.M. Jonathan Wu. 2017. High-order Local Normal Derivative Pattern 698 (LNDP) For 3d Face Recognition. IEEE International Conference on Image Processing (ICIP) 699 DOI 10.1109/ICIP.2017.8296795.

700

701

702

703

Heechul Jung, Sihaeng Lee, Junho Yim, Sunjeong Park, Junmo Kim. 2015. Joint Fine-Tuning in Deep Neural Networks for Facial Expression Recognition. IEEE International Conference on Computer Vision (ICCV) DOI 10.1109/ICCV.2015.341.

704

705

706

Amin Jourabloo and Xiaoming Liu. 2015. Pose-invariant 3D face alignment. IEEE International 707

Douglas M. Hawkins. 2004. The Problem of Overfitting. Journal of Chemical Information and

714 Computer Sciences 44:1-12.

P.J. Phillips, P.J. Flynn, T. Scruggs, K.W. Bowyer, J. Chang, K. Hoffman, J. Marques, J. Min, W. Worek. 2005. Overview of the face recognition grand challenge. CVPR DOI 10.1109/cvpr.2005.268.

716

Steve Lawrence and C. Lee Giles. 2000. Overfitting and Neural Networks: Conjugate Gradient 717 and Backpropagation. IJCNN DOI 10.1109/IJCNN.2000.857823.

719

L Perez and $\mathbf{J}$ Wang. 2017. The effectiveness of data augmentation in image classification using deep learning. arXiv 1712.04621:2-7.

720

SC Wong, A Gatt, and V Stamatescu. 2016. Understanding data augmentation for

722 classification: when to warp? IEEE 1:1-4 DOI 10.1109/dicta.2016.7797091.

723

724

725

Xiangyu Zhu, Xiaoming Liu, Zhen Lei, and Stan Z. Li. 2017. Face Alignment in Full Pose 726 Range: A 3D Total Solution. arXiv 1804.01005v1:9-10.

727

728

M. D. Zeiler and R. Fergus. 2014. Visualizing and understanding convolutional neural networks. 729 ECCV LNCS 8689 1: 818-833.

730 
731 Y. Sun, D. Liang, X. Wang, and X. Tang. 2015. DeepID3: Face Recognition with Very Deep

732 Neural Networks. ArXiv 1502.00873v1:2-4.

733

734

735

736

737

738

739

740

741

742

743

744

745

746

747

748

749

750

751

752

753

754

755

756

757

758

759

760

761

762

763

764

765

766

767

768

Zijun Cheng, Tianwei Shi, Wenhua Cui, Yunqi Dong, and Xuehan Fang. 2017. 3D face recognition based on kinect depth data. 4th International Conference on Systems and Informatics (ICSAI) 1:2-3 DOI 10.1109/ICSAI.2017.8248353.

Zhuoran Wu, Zhenjie Hou, Jian Zhang. 2017. Research on the 3D face recognition based on multi-class classifier with depth and point cloud data. IEEE Advanced Information Management, Communicates, Electronic and Automation Control Conference (IMCEC) 1:398-402 DOI 10.1109/IMCEC.2016.7867242.

Yi-bin Huang, Kang Li, Ge Wang, Min Cao, Pin Li, Yu-jia Zhang. 2015. Recognition of convolutional neural network based on CUDA Technology. arXiv 1506.00074:2-4.

Srishti Singh, Amrit Paul, and Dr. Arun M. 2017. Parallelization of Digit Recognition System Using Deep Convolutional Neural Network On CUDA. Third International Conference on Sensing, Signal Processing and Security (ICSSS) DOI 10.1109/SSPS.2017.8071623.

Amanda Song, Linjie Li, Chad Atalla and Garrison W.Cottrell. 2017. Learning to see faces like humans: modeling the social dimensions of faces. Journal of Vision 17(10):837 DOI $10.1167 / 17.10 .837$.

Hafiz Ahamed, Ishraq Alam, Md. Manirul Islam. 2018. HOG-CNN Based Real Time Face Recognition. Proc. International Conference on Advancement in Electrical and Electronic Engineering (ICAEEE) P-045:1-4 DOI 10.1109/ICAEEE.2018.8642989.

Patrick Flynn. 2006. Face Recognition Grand Challenge Biometrics Database (v2.0) License Agreement. In University of Notre Dame, Notre Dame. [Online]. Available: https://cvrl.nd.edu/media/django-summernote/2018-09-19/c7654649-5277-4d8c-b069-

483d8ffa3039.pdf DOI 10.6084/m9.figshare.9791951

Tang, H., Yin, B., Sun, Y., \& Hu, Y. 2013. 3D face recognition using local binary patterns. Signal Processing 93(8), 2190-2198. DOI 10.1016/j.sigpro.2012.04.002

Geetika Singh and Indu Chhabra. 2018. Effective and Fast Face Recognition System Using Complementary OCLBP and HOG Feature Descriptors with SVM Classifier. Journal of Information Technology Research 11:34-33.

Peer] Comput. Sci. reviewing PDF | (CS-2019:06:38908:1:3:NEW 6 Oct 2019) 
769

770 Delong Zhang, Min Zhang and Yongbo Liu. 2019. Three Dimension Face Recognition Based

771 on Gabor Transformation and Support Vector Machine. Journal of Applied Science and 772 Engineering, Vol. 22, No. 1, pp. 163170 (2019).

773

774 Jason Yosinski, Jeff Clune, Yoshua Bengio, and Hod Lipson. 2014. How transferable are

775 features in deep neural networks? The 27th International Conference on Neural Information 776 Processing Systems 2:3320-3328. 
Figure 1

The pre-processing of 3D face textures 
PeerJ Computer Science

\section{D Face Images (Input)}

\section{Face Detection}

\section{Face Alignment}

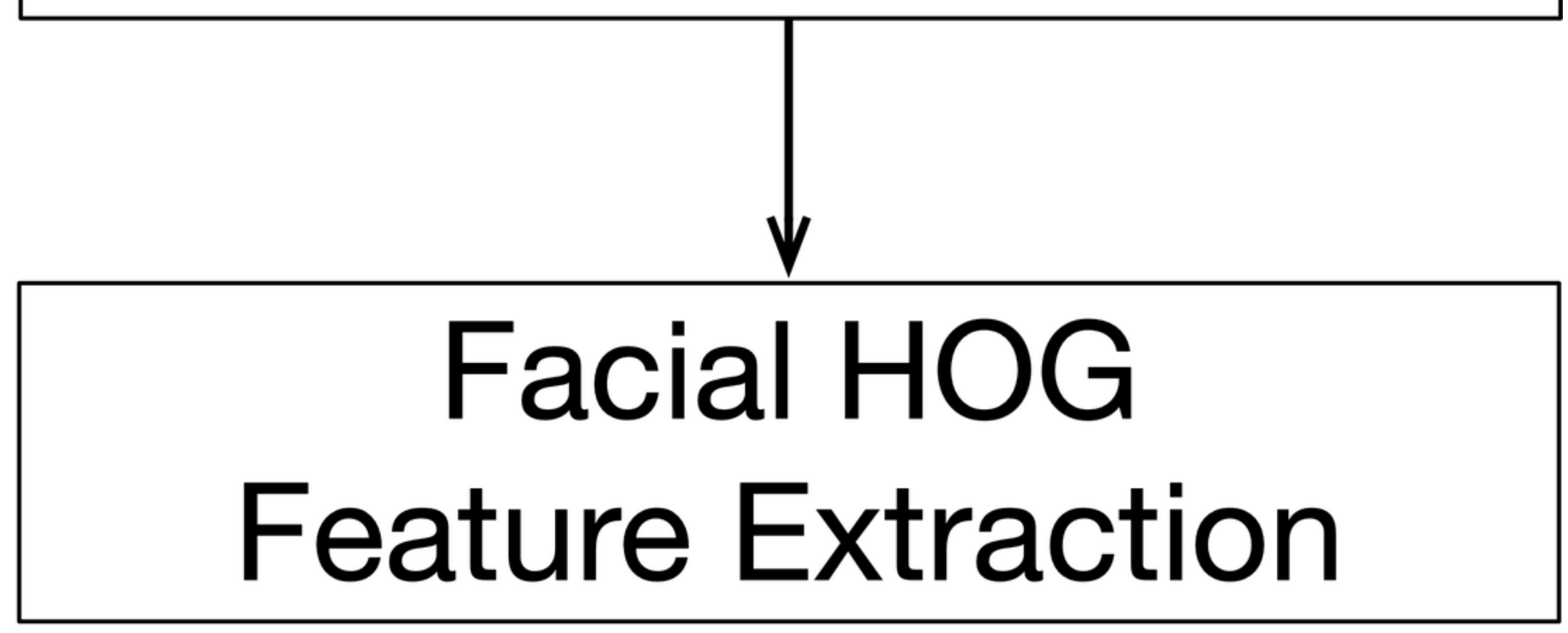

Peer] Comput. Sci. reviewing PDF | (CS-2019:06:38908:1:3:NEW 6 Oct 2019) 


\section{Figure 2}

Facial landmarks (68 key points) of Face Recognition Grand Challenge Version 2(FRGC v2.0)

\section{A}
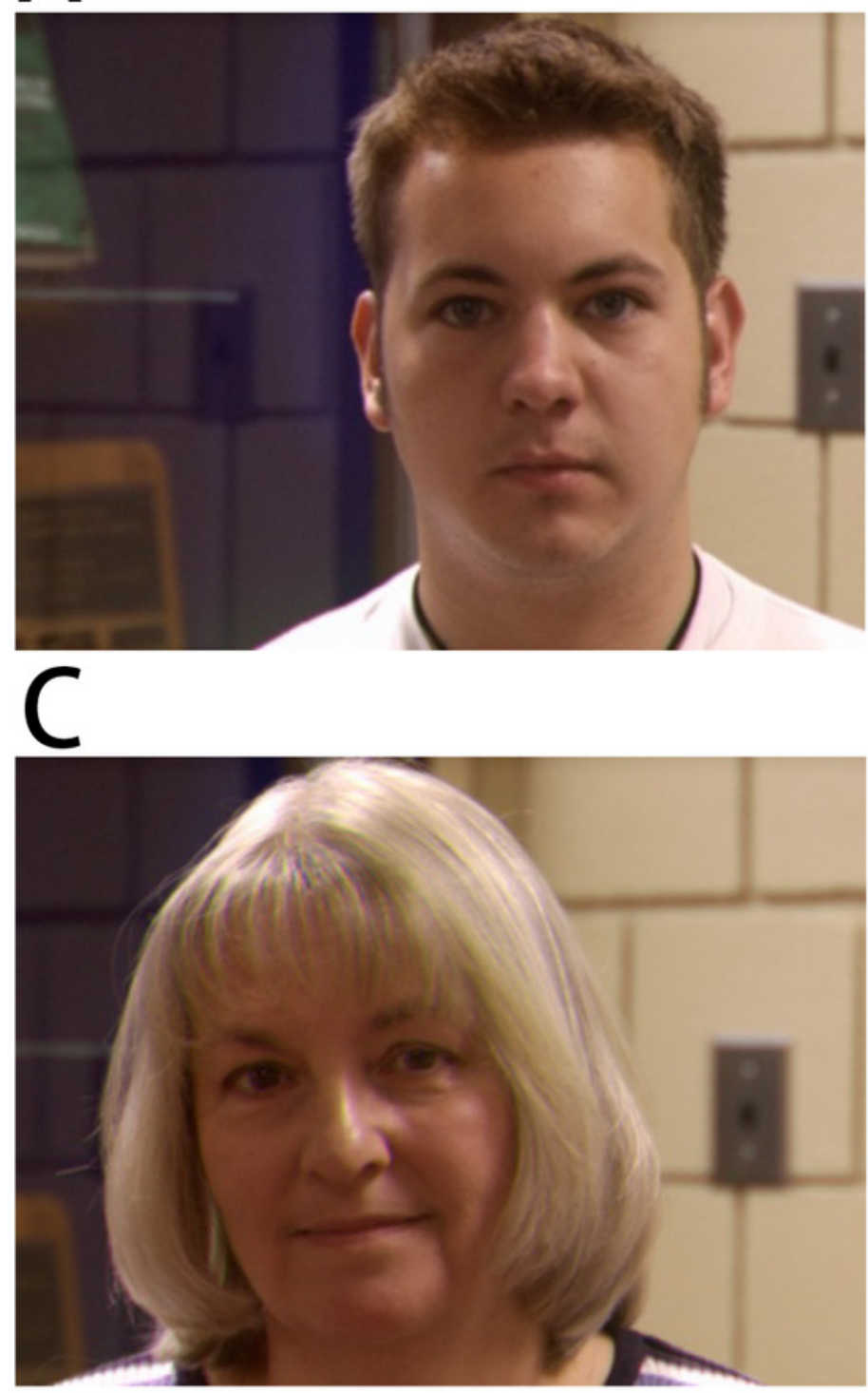

B
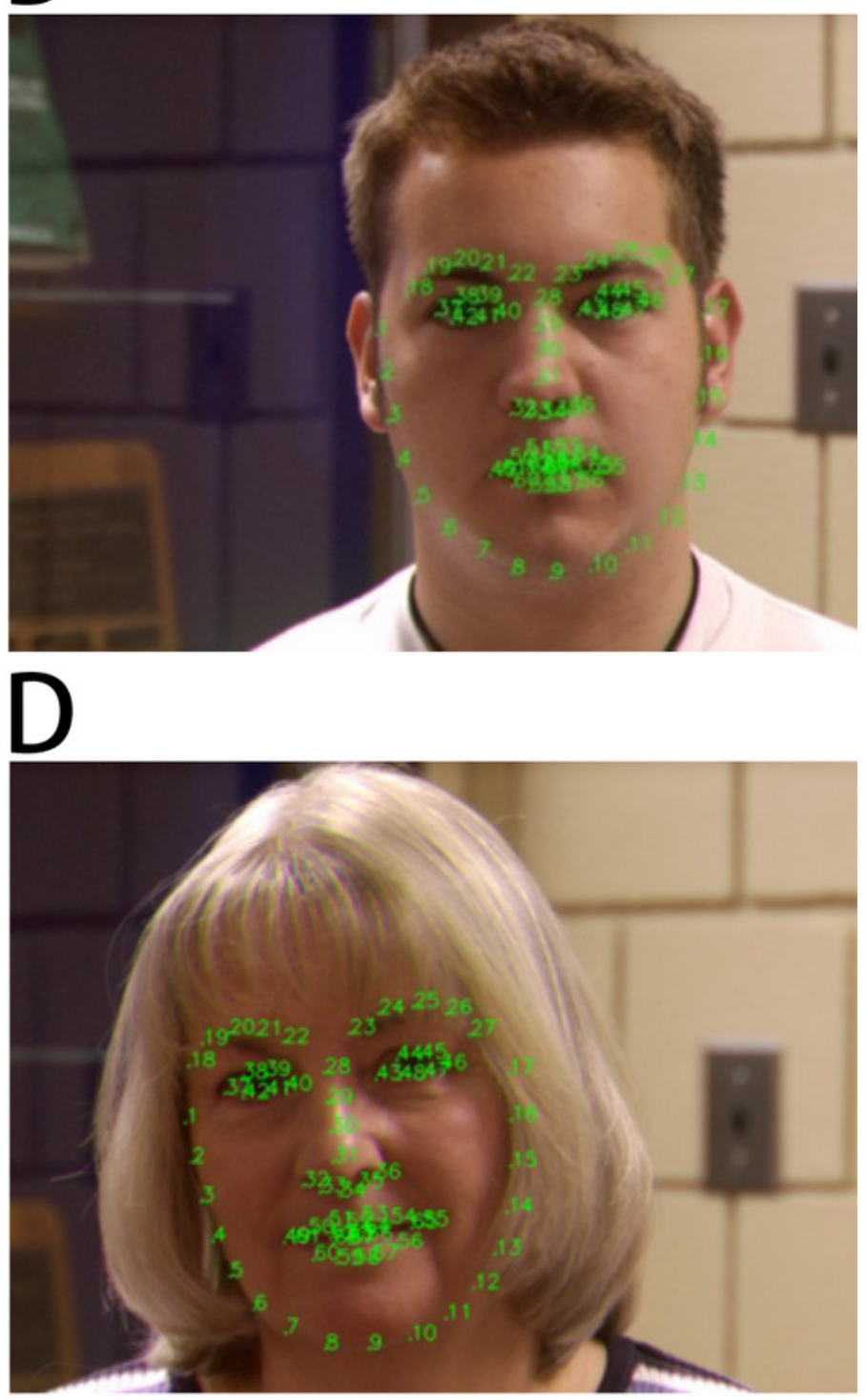
Figure 3

The processing of HOG feature extraction 


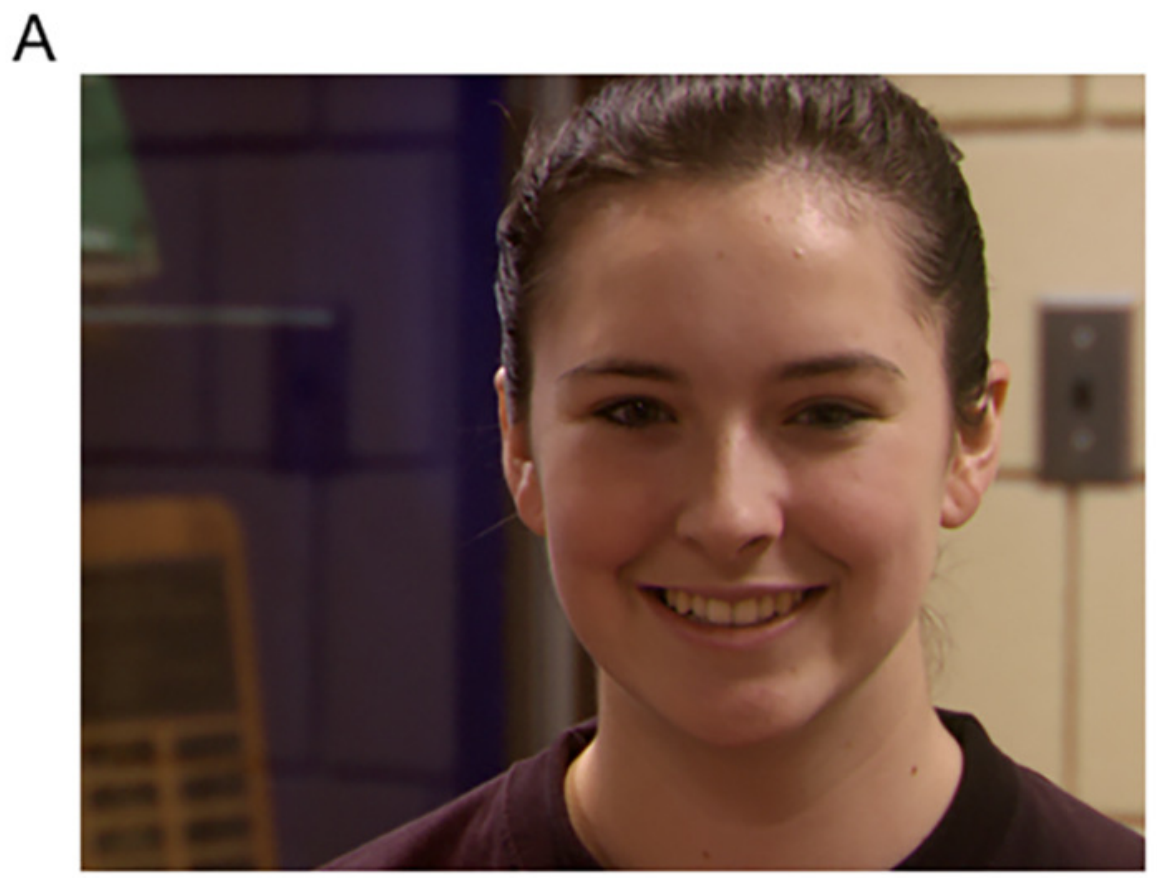

Original 3D face image

B

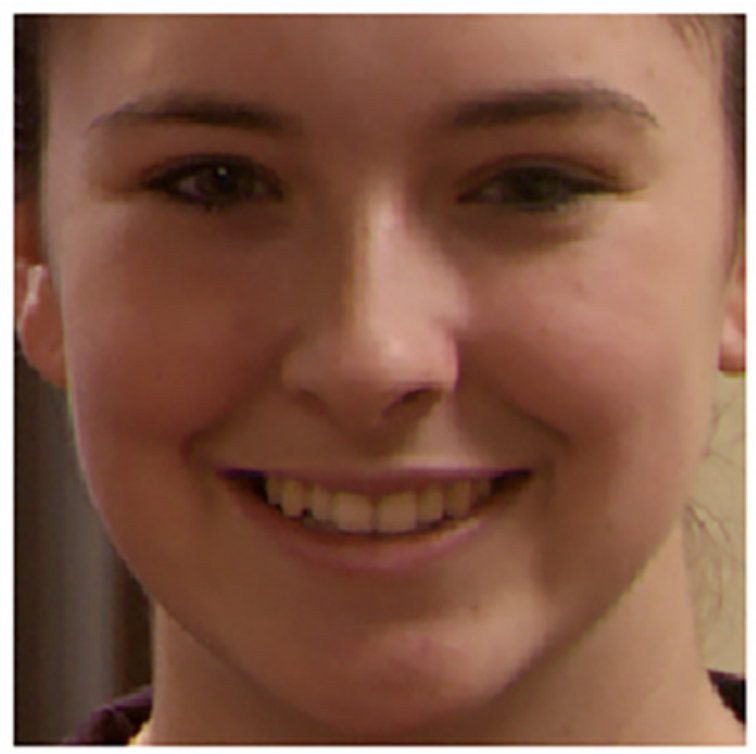

Input 3D face image
C

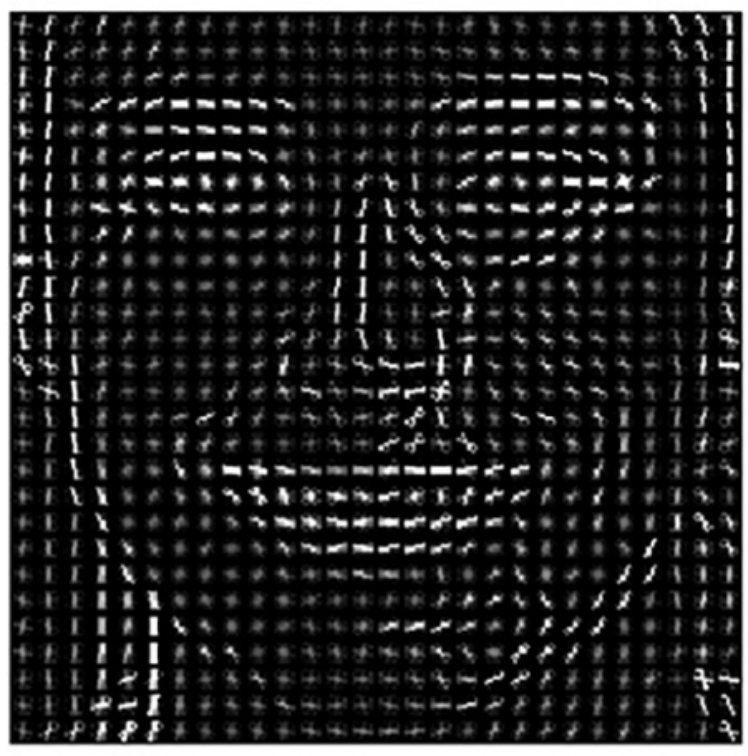

HOG feature extraction 


\section{Figure 4}

The HOG features of various 3D face images

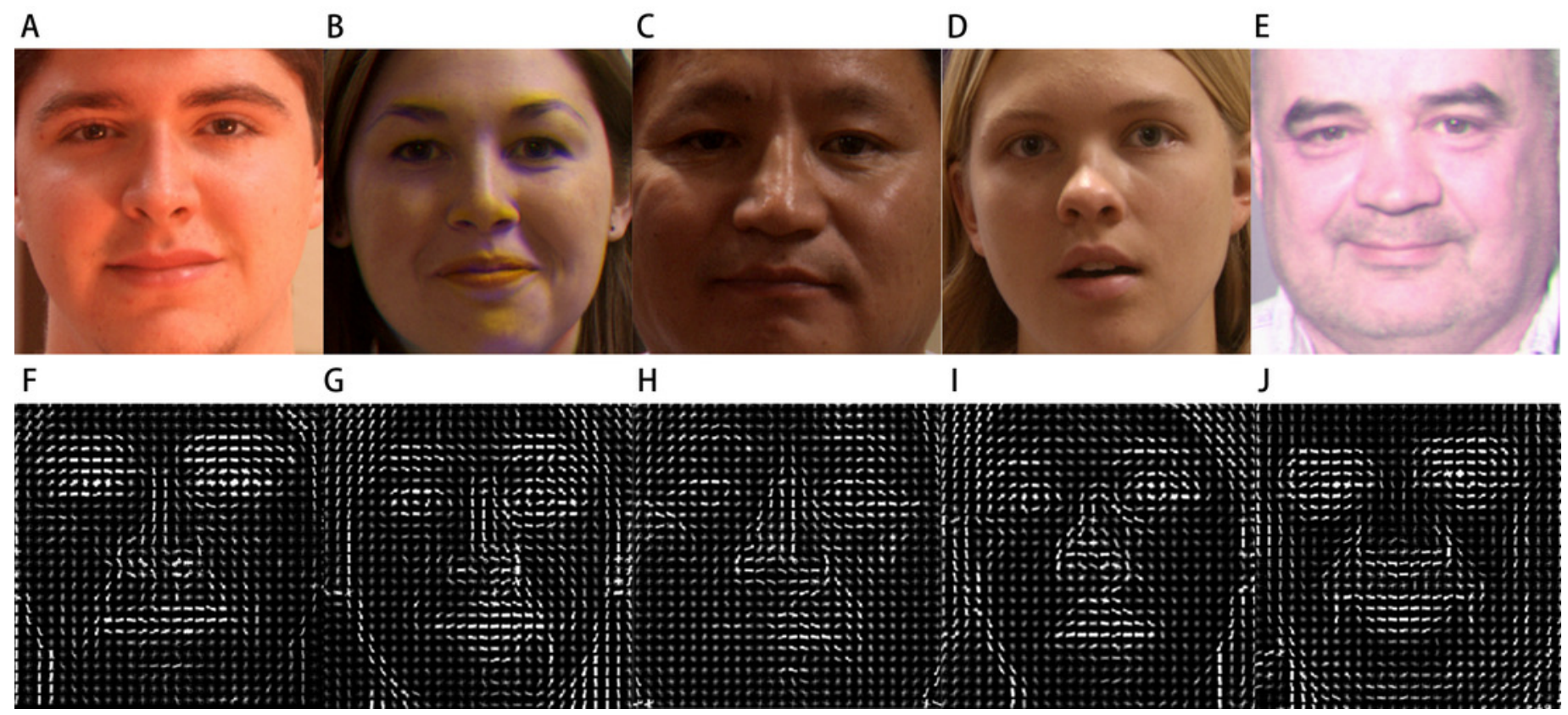


Figure 5

The architecture of ResNet model

A

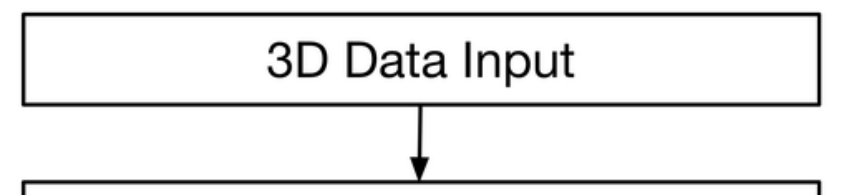

Convolution Layer $7 \times 7$, stride 2
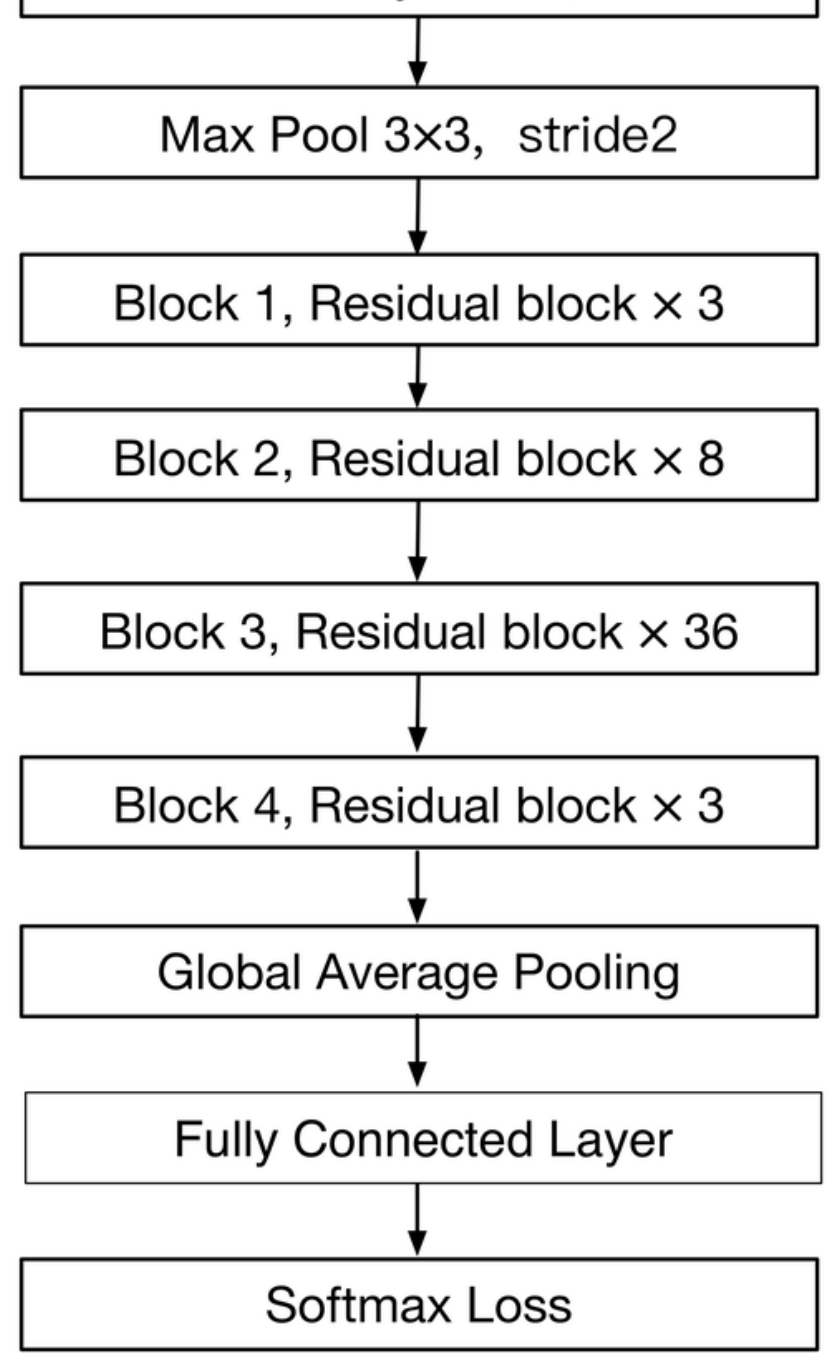

B

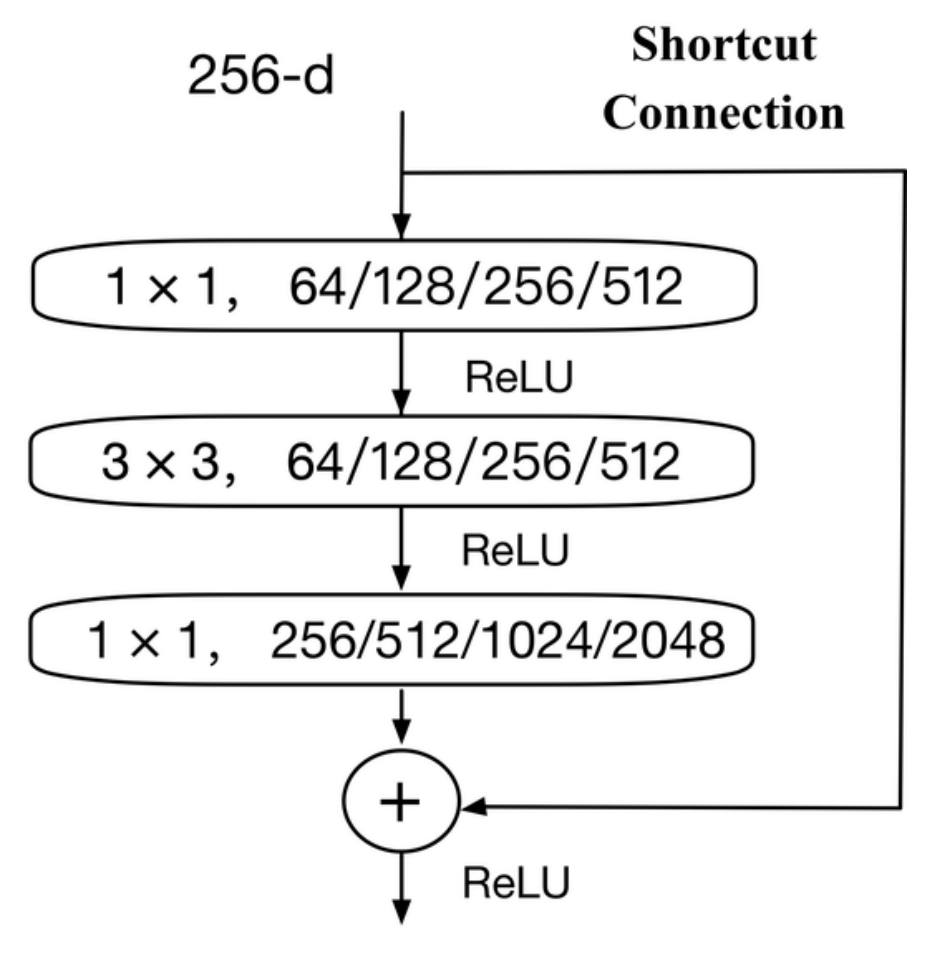


Figure 6

The fine-tuned ResNet neural network

\begin{tabular}{|c|c|c|}
\hline Structure & Output Size & Layers \\
\hline Conv layer 1 & $\begin{array}{l}\text { ( Input size: } \\
224 \times 224 \text { ) } \\
112 \times 112\end{array}$ & $\begin{array}{c}\text { ( Input channels: } 1) \\
7 \times 7,64, \text { stride } 2+\text { BatchNorm }\end{array}$ \\
\hline Conv layer 2 & $56 \times 56$ & $\begin{array}{l}\text { 3x3 max pool, stride } 2 \\
\begin{array}{l}1 \times 1,64 \\
3 \times 3,64 \\
1 \times 1,256\end{array} \quad \text { x3 + BatchNorm }\end{array}$ \\
\hline Conv layer 3 & $28 \times 28$ & $\begin{array}{l}1 \times 1,128 \\
3 \times 3,128 \\
1 \times 1,512\end{array} \quad \times 4+$ BatchNorm \\
\hline Conv layer 4 & $7 \times 7$ & $\begin{array}{l}1 \times 1,512 \\
3 \times 3,512 \\
1 \times 1,2048\end{array} \quad \times 3+$ BatchNorm \\
\hline & $1 \times 1$ & Adaptive Average Pooling \\
\hline & $1 \times 1$ & Fully Connected Layers \\
\hline & $1 \times 1$ & Softmax(466) \\
\hline \multicolumn{3}{|c|}{ Model Output } \\
\hline
\end{tabular}


Figure 7

The fine-tuned ResNet feature extraction operation

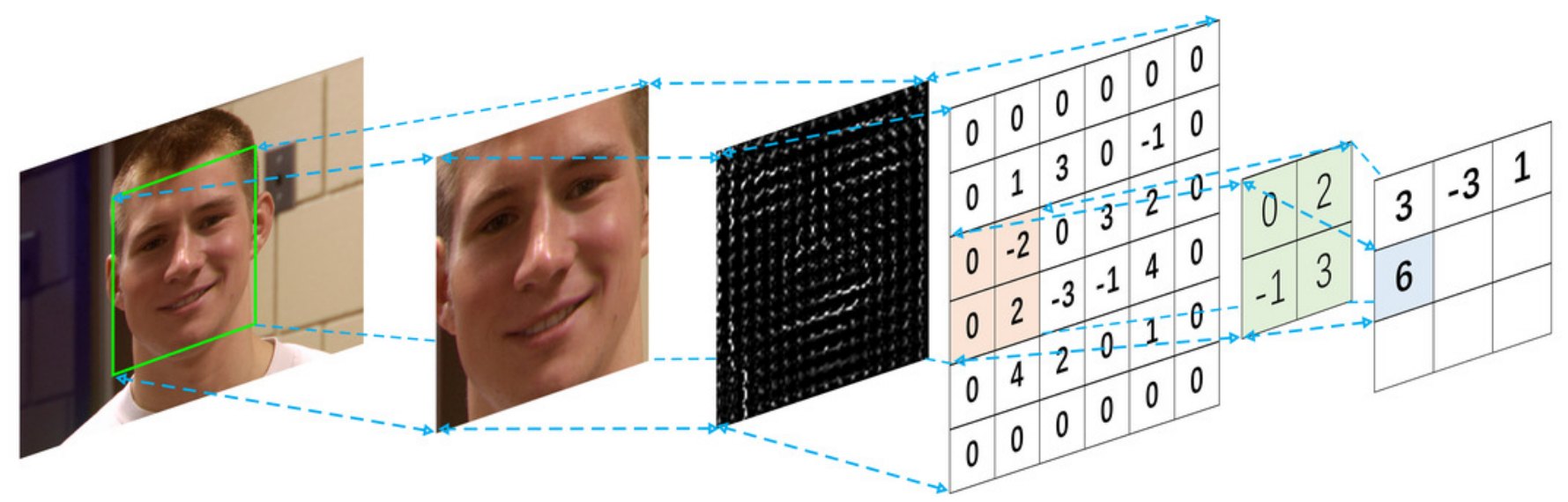


Figure 8

The accuracy rate of different layer numbers in the fine-tuning ResNet architecture

The performances are shown for fine-tuned ResNet-50 layer(A), fine-tuned ResNet-101 model(B), and Fine-tuned ResNet-152 model(C).
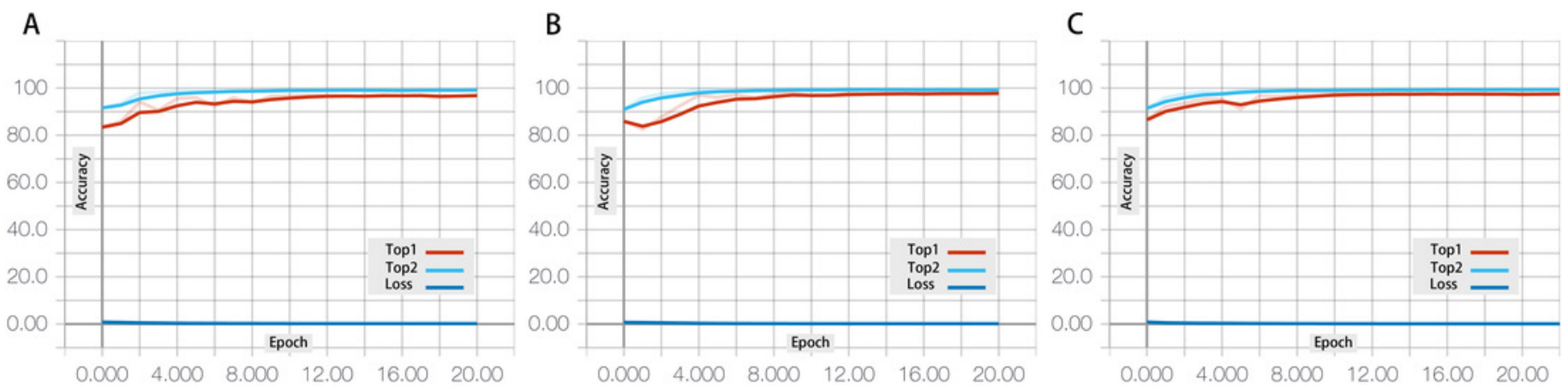


\section{Table $\mathbf{1}$ (on next page)}

Performance comparisons between the proposed method and state-of-the-art methods based on the FRGC-v2 dataset

Performance comparisons between the proposed method and state-of-the-art methods based on the FRGC-v2 dataset 
1 TABLE 2: Performance comparisons between the proposed method and state-of-the-art methods based 2 on the FRGC-v2 dataset.

3

4

5

6

\begin{tabular}{|c|c|c|c|}
\hline Method & Features & Classifier & Accuracy \\
\hline Huiying $\mathrm{Hu}$ et al. & Raw image & Custom CNN-2 & $85.15 \%$ \\
\hline S Sharma et al. & $\begin{array}{l}\text { Constrained } \\
\text { Local model }\end{array}$ & Custom CNN & $98 \%$ \\
\hline $\begin{array}{c}\text { Sima Soltanpour } \\
\text { et al. }\end{array}$ & $\begin{array}{c}\text { LNDP }^{3} x y z \\
\text { Based Normal } \\
\text { Component } \\
\text { Images }\end{array}$ & $\begin{array}{l}\text { SIFT-based } \\
\text { matching } \\
\text { Method }\end{array}$ & $98.10 \%$ \\
\hline $\begin{array}{c}\text { Proposed } \\
\text { Methodology }\end{array}$ & HOG features & $\begin{array}{c}\text { ResNet } 152 \text { layers } \\
\text { ResNet } 101 \text { layers } \\
\text { ResNet } 52 \text { layers } \\
\text { and } \\
\text { Fine-tuning }\end{array}$ & $\begin{array}{c}\text { Top1: } \mathbf{9 8 . 2 6 \%} \\
\text { Top2: } \mathbf{9 9 . 4 0 \%} \\
\text { Top1: } 97.77 \% \\
\text { Top2: } 99.40 \% \\
\text { Top1: } 97.02 \% \\
\text { Top2: } 99.12 \%\end{array}$ \\
\hline
\end{tabular}

7 